\title{
Beta cell regeneration after single-round immunological destruction in a mouse model
}

\author{
Jason M. Tonne • Toshie Sakuma • Miguel Munoz-Gomez • \\ Moustafa El Khatib • Michael A. Barry • Yogish C. Kudva • \\ Yasuhiro Ikeda
}

Received: 21 August 2014 / Accepted: 26 September 2014 / Published online: 23 October 2014

(C) The Author(s) 2014. This article is published with open access at Springerlink.com

\begin{abstract}
Aims/hypothesis Achieving a better understanding of beta cell regeneration after immunological destruction is crucial for the development of immunotherapy approaches for type 1 diabetes. In previous type 1 diabetes models, sustained immune activation eliminates regenerating beta cells, thus limiting the study of the regenerative capacity of beta cells upon immunological destruction. Here, we employed an adeno-associated virus 8 (AAV8) vector for beta cell-targeted overexpression of a foreign antigen to induce single-round immunological destruction of existing beta cells.

Methods Young and aged C57BL/6J mice were treated with AAV8 vectors expressing the foreign antigen luciferase. Islet inflammation and regeneration was observed at 3, 6, 10 and 22 weeks post-AAV delivery.

Results In young C57BL/6J mice, robust humoral and cellular immune responses were developed towards antigenexpressing beta cells, leading to decreased beta cell mass. This was followed by beta cell mass replenishment, along
\end{abstract}

J. M. Tonne and T. Sakuma contributed equally to this study.

Electronic supplementary material The online version of this article (doi:10.1007/s00125-014-3416-4) contains peer-reviewed but unedited supplementary material, which is available to authorised users.

J. M. Tonne · T. Sakuma • M. Munoz-Gomez • M. El Khatib •

Y. Ikeda $(\bowtie)$

Department of Molecular Medicine, Mayo Clinic College of

Medicine, 200 First Street SW, Rochester, MN 55905, USA

e-mail: ikeda.yasuhiro@mayo.edu

M. A. Barry

Department of Infectious Diseases, Mayo Clinic, Rochester, MN, USA

Y. C. Kudva

Division of Endocrinology, Mayo Clinic, Rochester, MN, USA with enhanced proliferation of insulin-positive cells, recruitment of nestin/CD34-positive endothelial cells, displacement of alpha cells and mobilisation of cytoplasmic neurogenin 3-positive cells. Mice with recovering beta cells showed normal or reduced fasting blood glucose levels and faster glucose clearance than controls. Although aged mice demonstrated similar responses to the treatment, they initially exhibited notable islet scarring and fluctuations in blood glucose levels, indicating that beta cell regeneration is slower in aged mice.

Conclusions/interpretation Our hit-and-run, beta cell-targeted antigen expression system provides an opportunity to monitor the impact of single-round immunological beta cell destruction in animals with diverse genetic backgrounds or ageing status.

Keywords AAV vector . Beta cell proliferation - Beta cell regeneration. Prediabetes. Type 1 diabetes

$\begin{array}{ll}\text { Abbreviations } \\ \text { AAV8 } & \text { Adeno-associated virus } 8 \\ \text { AAV9 } & \text { Adeno-associated virus 9 } \\ \text { CD } & \text { Cluster of differentiation } \\ \text { CTL } & \text { Cytotoxic T cell } \\ \text { EmGFP } & \text { Emerald green fluorescent protein } \\ \text { F4/80 } & \text { Murine macrophage marker } \\ \text { GFAP } & \text { Glial fibrillary acidic protein } \\ \text { GLP-1 } & \text { Glucagon-like peptide 1 } \\ \text { mIP2 } & \text { Mouse insulin promoter 2 } \\ \text { NES } & \text { Nestin } \\ \text { NGN3 } & \text { Neurogenin 3 } \\ \text { PDL } & \text { Partial ductal ligation } \\ \text { PDX1 } & \text { Pancreatic and duodenal homeobox 1 } \\ \text { p.i. } & \text { Post infection }\end{array}$




\section{Introduction}

In individuals susceptible to type 1 diabetes, exposure to environmental triggers is thought to induce abnormal activation of cellular and humoral immune responses against beta cells, leading to the initiation of beta cell destruction. The continuing destruction of beta cells results in the progressive reduction of insulin-secreting capacity [1]. At the time of clinical presentation of type 1 diabetes, approximately 60 $90 \%$ of the beta cells are estimated to be destroyed or dysfunctional [2]. Notably, several studies have demonstrated direct evidence of beta cell proliferation in early-onset type 1 diabetes $[3,4]$. The spontaneous recovery of beta cell function in some patients with early-onset type 1 diabetes also suggests that beta cells retain their regenerative capacity in type 1 diabetes [5]. Nevertheless, primarily because of the technical difficulties involved in monitoring beta cell mass in type 1 diabetic patients, the capacity for beta cell regeneration following immunological beta cell destruction has not been determined.

A better understanding of beta cell regeneration during or after immunological destruction may lead to the development of a curative type 1 diabetes therapy. Existing mouse models of type 1 diabetes, such as spontaneous or adoptive transferinduced type 1 diabetes, based on NOD mice, have been widely used to study type 1 diabetes pathogenesis. In NOD mice, an increased rate of beta cell proliferation is seen prior to a significant decline in beta cell mass, with the highest levels of proliferating beta cells ( $3 \% \mathrm{Ki} 67$ positive) being found at the onset of hyperglycaemia [6, 7]. Several combination therapies can reverse type 1 diabetes in NOD mice, indicating the possible recovery of endogenous insulin production in this type 1 diabetes model [8-12]. However, sustained autoimmunity towards beta cells generally results in the rapid elimination of regenerating beta cells, presenting a major barrier to monitoring beta cell regeneration capacity in type 1 diabetes models.

In this study, we employed an adeno-associated virus 8 (AAV8) vector for beta cell targeted, transient overexpression of a foreign antigen (firefly luciferase) and induced singleround immunological destruction of existing beta cells in young and aged mouse groups. Since non-integrating AAV vector DNAs are rapidly degraded in dividing cells [13], newly regenerated beta cells (from proliferation of either beta or progenitor cells) do not express the target antigen, thus enabling extensive characterisation of beta cell regeneration in the absence of sustained beta cell destruction.

\section{Methods}

For detailed methods, see the electronic supplementary material (ESM) Methods. A summary is given below.
Mice All studies were approved by the Mayo Clinic Institutional Animal Care and Use Committee. Male C57BL/6J mice were purchased from the Jackson Laboratory (Bar Harbor, Maine, USA). In vivo luciferase imaging was conducted as previously described [14]. Fasting blood glucose levels were monitored using the FreeStyle Lite Blood Glucose Monitor (Abbott Laboratories, Chicago, Illinois, USA). Glucose tolerance tests were conducted by fasting the mice for 4-5 h, followed by i.p. delivery of $2 \mathrm{~g} / \mathrm{kg}$ D-glucose in a 30\% PBS solution. Blood glucose was checked after $0,30,60,90$ and $120 \mathrm{~min}$.

Cells 293T cells were maintained in DMEM supplemented with $10 \%$ calf serum, $50 \mathrm{U} / \mathrm{ml}$ penicillin and $50 \mu \mathrm{g} / \mathrm{ml}$ streptomycin. Cells were cultured at $37^{\circ} \mathrm{C}$ with $5 \% \mathrm{CO}_{2}$.

Plasmids The $1.13 \mathrm{kbp}$ mouse insulin 2 promoter (mIP2) [15] was PCR-amplified from mouse genomic DNA using the primers 5'-GCCACACGCGTCCCTCCTCTTGCATTTCA AAT-3' and 5'-TCCACAGGATCCTGTTGAAACAATAA CCTGGAA-3'. pAAV-mIP2-Luc vectors were generated by replacing the cytomegalovirus promoter of the pAAV-CMVLuc plasmid [14] with the mIP2 sequence (bold text indicates Mlu1 and BamH1 restriction sites). The pAAV-mIP2-emerald green fluorescent protein (EmGFP) vector was cloned by replacing the luciferase transgene with EmGFP cDNA.

AAV8 vectors Helper-free AAV8 vector stocks were produced as previously described [16]. The pRC-2/8 AAV8 capsidexpressing plasmid was kindly provided by J. Wilson (University of Pennsylvania, Philadelphia, PA, USA).

$A A V$ vector administrations Mice received an i.p. injection of AAV8 vectors at a final dose of $2 \times 10^{11}$ genome copies per mouse.

Detection of anti-luciferase antibody Luciferase-expressing and control $293 \mathrm{~T}$ cell lysates were separated by SDS-PAGE. Plasma samples were used as the primary antibody in western blotting.

IFN- $\gamma$ ELISpot assay The assay was performed using the IFN- $\gamma$ ELISpot Mouse Set (BD Pharmingen, San Diego, CA, USA). Splenocytes were added to duplicate wells at a density of $0.1 \times 10^{6}, 0.5 \times 10^{6}$ or $1 \times 10^{6}$ cells per well along with DMEM-10 with or without $2.0 \mu \mathrm{g} / \mathrm{ml}$ ovalbumin peptide or $2.0 \mu \mathrm{g} / \mathrm{ml}$ firefly luciferase epitope peptide. The ovalbumin T cell-reactive peptide sequence (SIINFEKL) [17] and firefly luciferase T cell-reactive epitope (LMYRFEEEL) [18] were synthesised by GenScript (Piscataway, New Jersey, USA).

Immunostaining All immunostaining was conducted as previously described [16]. Antibodies and the 
concentrations used for immunocytochemistry are described in the ESM Methods.

Insulitis scoring The insulitis score was determined by following established criteria [19]. Three $7 \mu \mathrm{m}$ thick whole headto-tail pancreatic sections (each $200 \mu \mathrm{m}$ in depth) were collected per animal and co-stained with anti-insulin and anticluster of differentiation 45 (CD45) antibodies, with DAPI labelling.

Insulin- and glucagon-positive area analysis Pancreatic sections were prepared and the insulin-positive area was quantified by using the formula: Percentage insulin-positive area= insulin-positive area/total tissue area $\times 100$ [16].

Mouse pancreatic RNA extraction Pancreases were isolated and three tissue sections ( $\sim 20 \mathrm{mg})$ were immediately processed using an RNeasy Plus Mini Kit (Qiagen, Limburg, Netherlands).

RT-PCR and quantitative PCR One microgram of total RNA was used to synthesise cDNA (EcoDry Premix, Clontech Laboratories, Mountain View, California, USA). Quantitative PCR was conducted using SYBR green-based expression analysis in QuantiTect Primer Assays (Qiagen). Firefly luciferase expression was determined using SYBR green quantitative PCR with primers based on a $140 \mathrm{bp}$ segment of the luciferase gene: Forward FFLuc_qPCR_F, 5'GCTAT TC T GAT TACACCCGAGG-3'; Reverse FFLuc_qPCR_R 5'-TCCTCTGACACATAATTCGCC-3'.

Sample size and statistical analysis Groups were compared by unpaired Student's $t$ test, and data are expressed as means \pm SEM. Significance was set at $p<0.05$.

\section{Results}

Systemic administration of AAV8 vectors containing the murine insulin 2 promoter facilitates beta cell-targeted transgene expression AAV8 vectors were engineered to express firefly luciferase under the control of an internal promoter of the mouse Ins 2 gene (Fig. 1a, ESM Fig. 1a, b). Mice were i.p. injected with the AAV8 vectors, and luciferase expression was monitored 2 weeks post infection (p.i.). AAV vectors containing $\mathrm{mIP} 2$ [15] exhibited potent, pancreas-restricted luciferase expression (Fig. 1b). When the mIP2-AAV8 vector was used to deliver the EmGFP gene (Fig. 1a), i.p. administration of AAV8 vectors $\left(2 \times 10^{11}\right.$ genome copies/mouse $)$ resulted in selective EmGFP expression in insulin-positive beta cells (Fig. 1c), demonstrating beta cell-specific transgene expression via the mIP2-AAV8 vector system. When
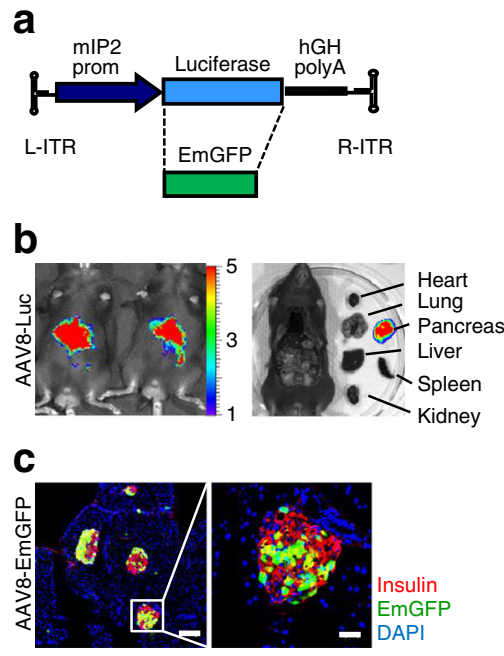

Fig. 1 Systemic administration of AAV8 vectors containing mIP2 facilitates beta cell-targeted transgene expression. (a) Schematic representation of AAV vectors. L-ITR, left inverted terminal repeat; R-ITR, right inverted terminal repeat; prom, promoter. (b) AAV8-mediated luciferase expression was assessed 14 days after i.p. injection (control $n=3$, AAV8Luc $n=3$ ). Luminescence scale bar, $\times 10^{4}$. (c) EmGFP expression was assessed in pancreatic sections EmGFP $(n=2)$. Selective EmGFP expression (green) was observed in insulin-positive beta cells (red), but not in acinar cells. Nuclei were counterstained with DAPI (blue). Scale bars, $100 \mu \mathrm{m}$

EmGFP transduction efficiency was assessed from 15 random islets, the proportion of EmGFP-positive islet mass reached up to $66 \%$ (average $47.8 \%$ ), relative to the insulinpositive area $(n=2)$.

Beta cell targeted luciferase expression induces luciferasespecific humoral and cellular immune responses Five-weekold mice ( $n=4$ per time point) were i.p. injected with the luciferase-expressing mIP2-AAV8 vector (AAV8-Luc). AAV8-Luc treated and control (PBS-injected) mice were monitored for luciferase expression (Fig. 2a). Luciferase signals in the pancreas were observed for 10 weeks p.i. Expression of beta cell-specific luciferase reached a peak at around 3 weeks p.i., followed by a dramatic decline in luminescent signals (Fig. 2a). This correlated well with a marked decline in luciferase transcripts in the pancreas (Fig. 2b). To investigate luciferase-specific adaptive immune responses, plasma samples and splenocytes were harvested and analysed at 3, 6, 10 and 22 weeks p.i. Three out of four mice showed weak-to-moderate levels of circulating antibodies against luciferase by 3 weeks p.i., whereas all mice showed strong humoral immunity against luciferase by 6 and 10 weeks p.i. (Fig. 2c). AAV capsid-specific antibody was not detected in serum (Fig. 2d). Beta cell-targeted luciferase overexpression also induced potent cellular immunity. An ELISpot assay revealed a luciferase-specific cytotoxic $\mathrm{T}$ lymphocyte (CTL) response at 6 weeks p.i. (Fig. 2e). The CTL 
a
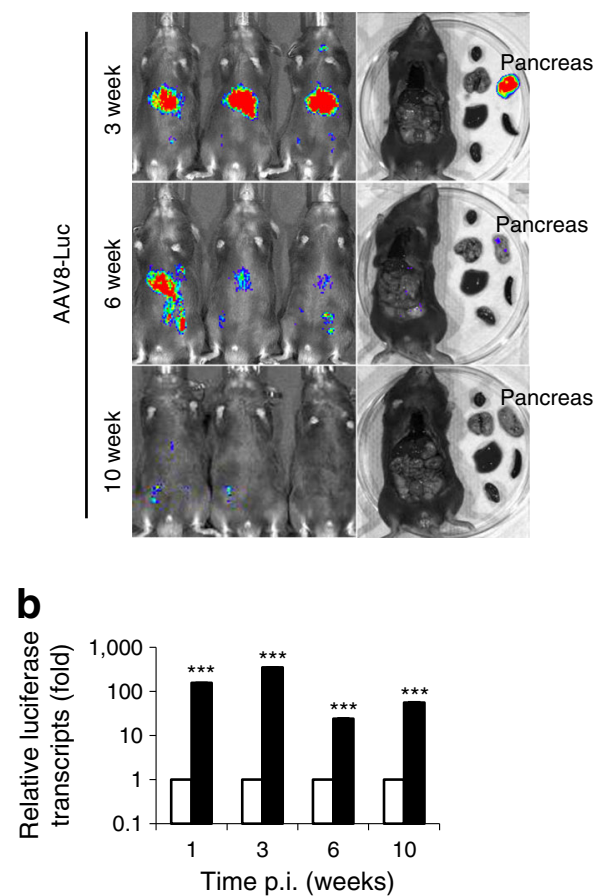

C
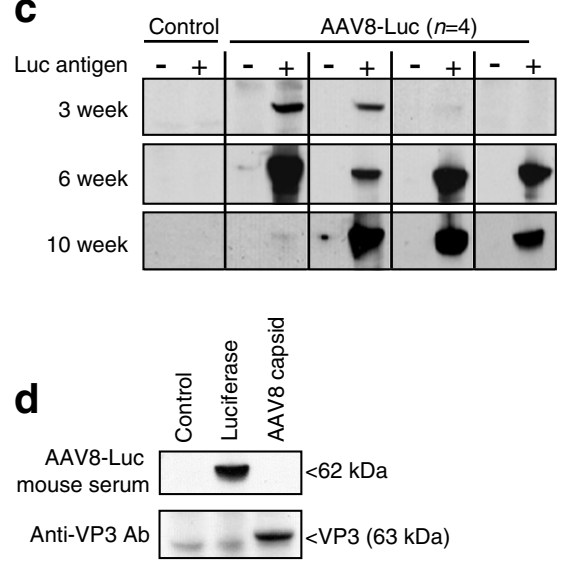

e Control $\frac{\text { AAV8-Luc }}{3 w e c k}$
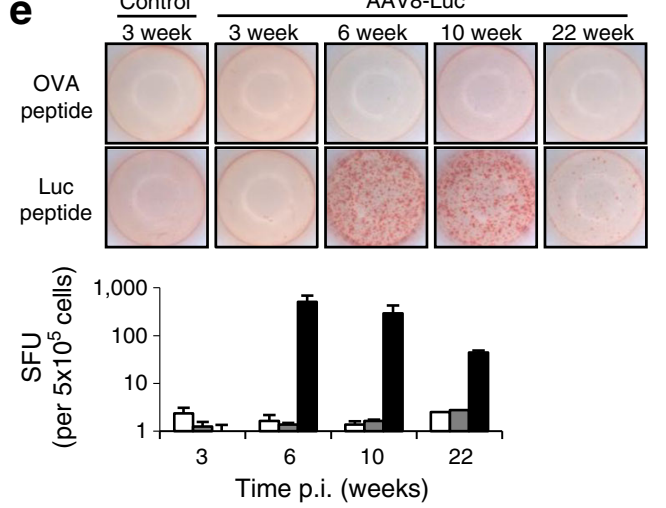

f

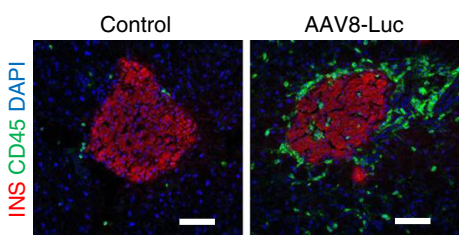

\begin{tabular}{rrrrrrrrr}
\hline & \multicolumn{3}{c}{ Control } & & \multicolumn{3}{c}{ AAV8-Luc } \\
\cline { 2 - 4 } \cline { 6 - 7 } Animal ID & 1 & 2 & 3 & & 5 & 6 \\
\hline Total islets counted & 13 & 18 & 21 & & 23 & 30 & 24 \\
Ave. intra-islet CD45+ & 0.5 & 0.5 & 0.1 & & 4.7 & 2.0 & 7.8 \\
Ave. peri-islet CD45+ & 4.3 & 4.4 & 2.8 & & 36.3 & 30.2 & 51 \\
Islets >15 CD45+ & 0 & 1.0 & 0 & 20 & 17 & 23 \\
\% islets >15 CD45+ & 0 & 5.6 & 0 & 87.0 & 56.7 & 95.8 \\
\hline
\end{tabular}

g

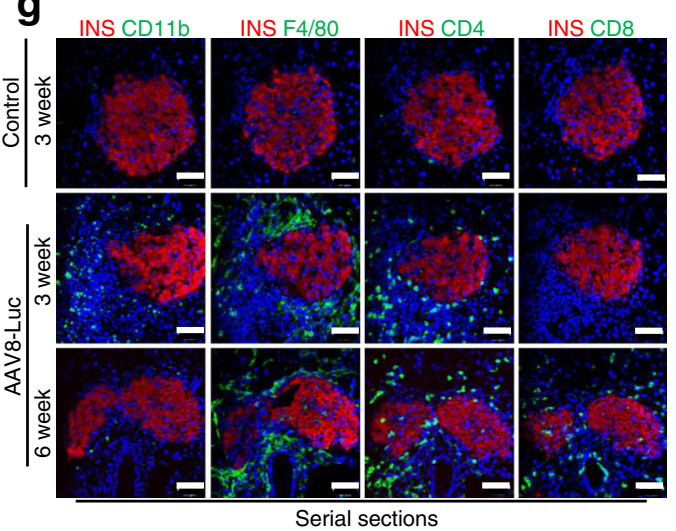

infected mice that react to the luciferase epitope peptide LMYRFEEEL. Ovalbumin (OVA) peptide was used as a negative control (grey bars). Splenocytes were analysed after 3, 6, 10 and 22 weeks. Representative wells and the means \pm SEM of spot-forming units (SFU) from four mice per group per time point are shown. Logarithmic scale, log base 10. (f) Representative islets used for insulitis scoring with an anti-CD45 antibody at 4 weeks p.i. The table shows the number of random islets counted and the average (Ave.) intra- and peri-islet CD45 cells ( $n=3$ per group). (g) Serial pancreatic sections were stained with antibodies against the immune cell markers CD11b, F4/80, CD4 and CD8 (green). Nuclei were counterstained with DAPI (blue). Scale bars, $50 \mu \mathrm{m}$. INS, insulin; Luc, luciferase

High-grade islet infiltration by CD4- and CD8-positive T cells and macrophages was observed in a patient with recentonset type 1 diabetes [20]. Immunohistochemical analysis of 
the leucocyte common antigen CD45 was performed to detect inflamed islets, defined as an islet containing more than 15 CD45-positive cells [19]. We found $0-5.6 \%$ and $57-96 \%$ islets with peri-insulitis or insulitis in control and AAV8-Luc-treated mice, respectively, at 4 weeks p.i. (Fig. 2f). Further analysis demonstrated that pancreatic islets were surrounded by CD11b-positive innate immune cells, murine macrophage marker (F4/80)-positive macrophages and CD4-positive helper T cells as early as 3 weeks p.i. At 6 weeks p.i., there were fewer CD11b-positive cells, but islets were infiltrated with CD8- positive CTLs, as well as F4/80- and CD4-positive cells (Fig. 2g). The timing of CTL infiltration into the islets correlated with the induction of the luciferase-targeted CTLs (Fig. 2e).

Beta cell targeted immune response results in a transient beta cell mass decline followed by robust beta cell regeneration We next measured the total insulin-positive area at multiple time points (Fig. 3a, b, ESM Fig. 2a). A significant decline in beta cell mass was observed 6 weeks post AAV delivery. The correlation between reduced beta cell mass and
Fig. 3 Beta cell-targeted immune responses result in a transient reduction in beta cell mass followed by robust beta cell regeneration. (a) Pancreatic islets were visualised by staining with anti-glucagon (GCG) and antiinsulin (INS) antibodies. Images of the islets at 6 weeks p.i. are shown. Scale bar, $100 \mu \mathrm{m}$. (b) Insulin-positive area as a percentage of the total pancreatic area in treated and control mice $(n=4$ for each time point; $\left.{ }^{*} p<0.05,{ }^{* *} p<0.01\right)$. (c) Representative pancreatic islets from AAV8-Luc treated or control mice stained with antiinsulin and anti-Ki67 antibodies. Insulin-positive and Ki67positive islet cells from AAV8Luc treated or control mice were counted, and the percentage of insulin/Ki67 double-positive cells was determined. Data are presented as means \pm SEM. Scale bar, $100 \mu \mathrm{m}$. ${ }^{*} p<0.05$ vs control. (d) Groups of single insulinpositive and Ki67-positive cells detected at 10 weeks p.i. are shown. Scale bars, $100 \mu \mathrm{m}$. (e) Fasting blood glucose levels were monitored ( $n=4$ per group). ${ }^{*} p<0.05, * * p<0.01$. (f) Glucose tolerance test on control and treated mice ( $n=4$ per group) at 6 weeks p.i. $* p<0.05$. (g) Fasting plasma insulin levels were determined by ELISA at 3 and 10 weeks p.i. $(n=3$ per group per time point). (b), (c), (e-g) white bars/squares, saline controls; black bars/squares, AAV8-Luc treatment
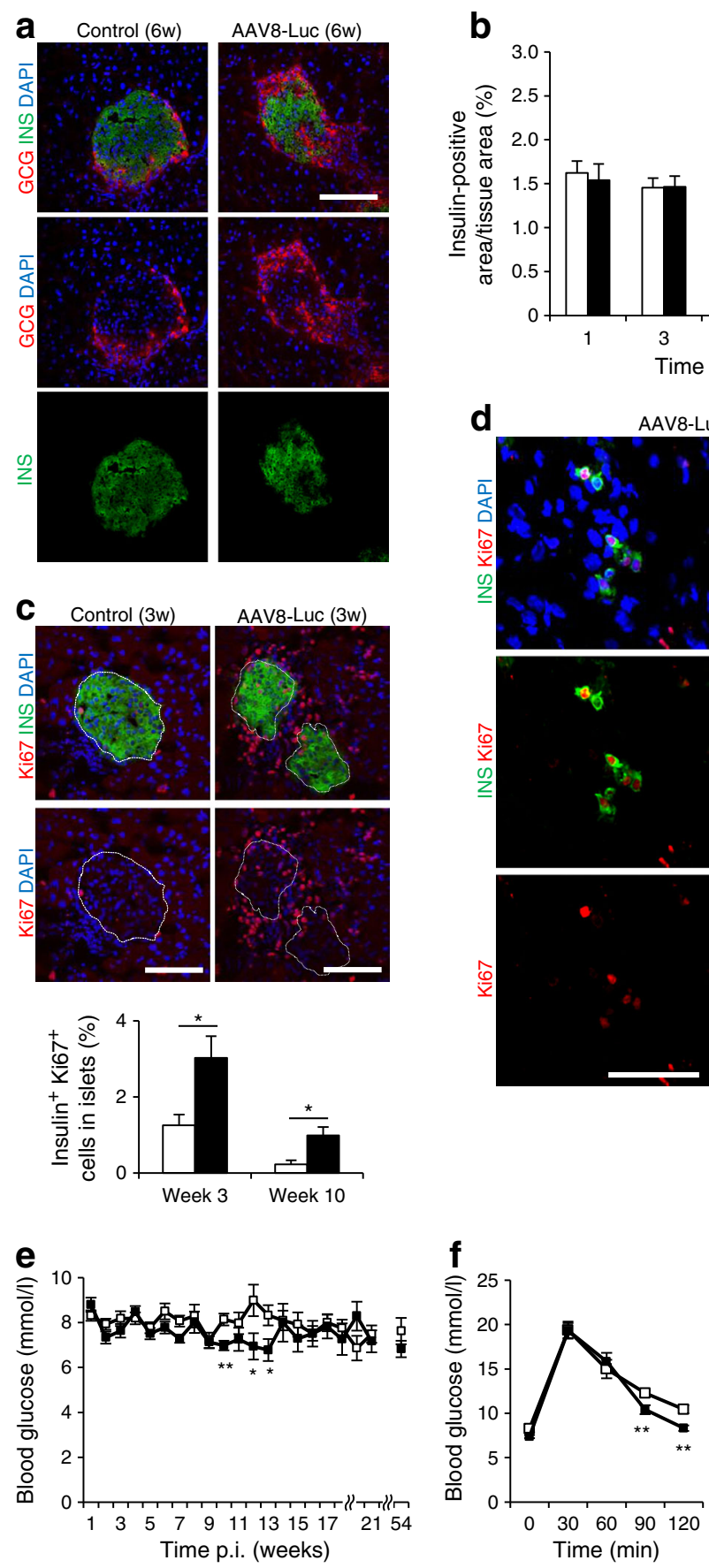
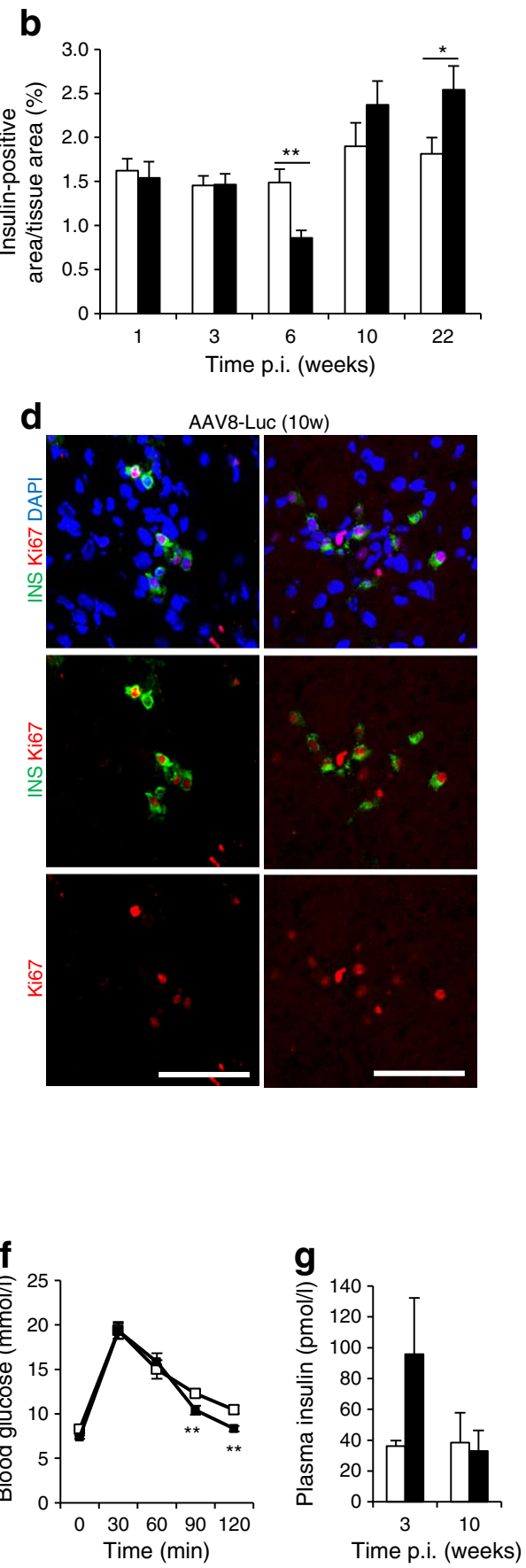

Time p.i. (weeks) 
the induction of beta cell-targeted CTLs supports the widely accepted concept that CD8-positive CTL-mediated beta cell killing is probably a major mechanism of beta cell destruction [2]. At 10 weeks p.i., the beta cell mass recovered to normal levels. By week 22, there was a slight increase in beta cell mass in damaged islets over control (Fig. 3b).

To assess beta cell regeneration, we counted the number of insulin-positive, Ki67-positive cells within islets (Fig. 3c, upper panels). Beta cell proliferation increased in damaged and recovering islets at 3 weeks and 10 weeks p.i. by 2.4-fold $(3.03 \%)$ and 4.4 -fold (0.99\%), respectively (Fig. 3c). In addition, AAV-Luc-treated mouse pancreases frequently showed groups of single insulin-expressing cells at 10 weeks p.i., some of which were also positive for the Ki67 proliferation marker (Fig. 3d). These observations indicate that robust beta cell regeneration occurs after immunological destruction in young mice. Although we found increased apoptotic TUNELpositive cells in and around inflamed islets at 3 and 6 week p.i., most TUNEL-positive cells were not insulin positive (ESM Fig. 2b).

In the presence of immunological beta cell damage, AAVLuc-transduced mice remained normoglycaemic: fasting blood glucose levels remained $<10 \mathrm{mmol} / \mathrm{l}$ (Fig. 3e). The only trend we observed was a moderate decline in fasting blood glucose levels between weeks 8 and 13. This observation was also made following expression of another foreign antigen, ovalbumin, via the mIP2-AAV8 vector (ESM Fig. 3a). Body weights were the same for mice in both groups (ESM Fig. 3b). Upon glucose stimulation at 3, 6, 10 and 54 weeks post-AAV delivery, treated mice responded similarly to controls, but with slightly faster glucose clearance at 6 weeks p.i. (Fig. 3f, ESM Fig. 3c). Additionally, transient elevations in circulating insulin and glucagon-like peptide 1 (GLP-1) were observed during the immunological attack that occurred at 3 weeks p.i., but not during beta cell recovery at 10 weeks (Fig. $3 \mathrm{~g}$ and ESM Fig. 3d).

\section{Displacement of glucagon-positive cells and activation of} neurogenin-3-positive cells following immunological beta cell damage Increased glucagon production and secretion are often associated with insulin deficiency in type 1 diabetes patients [21], while the conversion of adult alpha cells to beta cells has been reported following extreme beta cell loss [22, 23]. We assessed changes in the glucagon-expressing alpha cell population following immunological beta cell destruction. Control islets showed a normal distribution of alpha cells around the perimeter of insulin-positive islets (Fig. 4a). In contrast, following immunological beta cell injury, we frequently observed an irregular distribution or displacement of glucagon-positive cells around damaged islets (Fig. 4a, ESM Fig. 2a). The relative proportion of alpha cells was frequently increased in inflamed islets at 6 weeks p.i. (Figs $3 a$ and $4 a$ ), with a significant increase in the glucagon-positive area relative in AAV8-Luc-treated mice $(1.67 \pm 0.39 \%$ and $4.70 \pm$
$0.91 \%$ in control and treated mice, respectively). Alpha cell displacement was also evident at 10 weeks p.i., with the frequent appearance of single glucagon-positive cells outside recovering islets (Fig. 4a).

We also assessed expression of neurogenin 3 (NGN3), the beta cell progenitor marker. Nuclear NGN3 expression was evident in all control islets up to 6 weeks (up to age 12 weeks; Fig. 4b, ESM Fig. 4a). Nuclear NGN3 signals frequently colocalised with the mature beta cell marker pancreatic and duodenal homeobox 1 (PDX1) (Fig. 4b right panel, ESM Fig. 4a), while nuclear NGN3 expression was found in glucagon-positive alpha cells (ESM Fig. 4b). Strong cytoplasmic NGN3 localisation was infrequently observed in cells around control islets, especially those adjacent to pancreatic ducts (Fig. 4c, ESM Fig. 4c). These cytoplasmic NGN3positive cells did not express insulin or PDX1.

Imaging of NGN3-positive cells in AAV vector-treated mouse pancreases showed a general decrease in nuclear NGN3 expression in beta cells and a marked increase in cytoplasmic NGN3-positive cells in and around the damaged islets (Fig. 4d, left panel), especially at 6 weeks p.i. (Fig. 4d, middle panel; ESM Figs 5 and 6). At 10 weeks p.i., cytoplasmic NGN3-positive cells were frequently found between the recovering islets and adjacent ducts (Fig. 4d, right panel, Fig. 4e, f; ESM Figs 6 and 7). This was accompanied by induction of PDX1 expression in these ductal cells (Fig. 4e, f). In contrast to control islets, glucagon-positive cells were also found at this location (between ducts and islets) at 10 weeks p.i. (Fig. 4f, ESM Fig. 7), although cytoplasmic NGN3-positive cells and glucagon-expressing cells formed distinct populations (Fig. 4g). Single insulin-positive cells were often found together with cytoplasmic NGN3-positive cells at 10 weeks p.i. (Fig. 4h).

To confirm our immunohistochemistry results, we conducted quantitative RT-PCR analysis for key pancreatic genes (Fig. 4i-p). Changes in expression of the major rodent insulin gene, Ins2, correlated well with changes in beta cell mass in the pancreas. Pancreatic expression of Gcg, $P d x 1$ and $N k x 6.1$ was comparable between treated and untreated mice. In contrast, expression of Ngn3, MafA and NeuroD1, transcription factors critical for beta cell development, was significantly elevated 6 and 10 weeks after AAV administration.

Recruitment of NES- and CD34-positive vascular endothelial cells to damaged islets Nestin (NES) and CD34 are key surface markers of neuronal and haematopoietic stem cells $[24,25]$. Previous studies also identified NES-expressing cells as potential pancreatic progenitor cells [26]. We therefore assessed changes in NES and CD34 expression in the pancreas. Control islets consistently showed NES-positive cells within the islets and a diffuse distribution around the islet exterior (ESM Fig. 8a, left panel). During immunological beta cell damage, there was a dramatic increase in NES-positive 

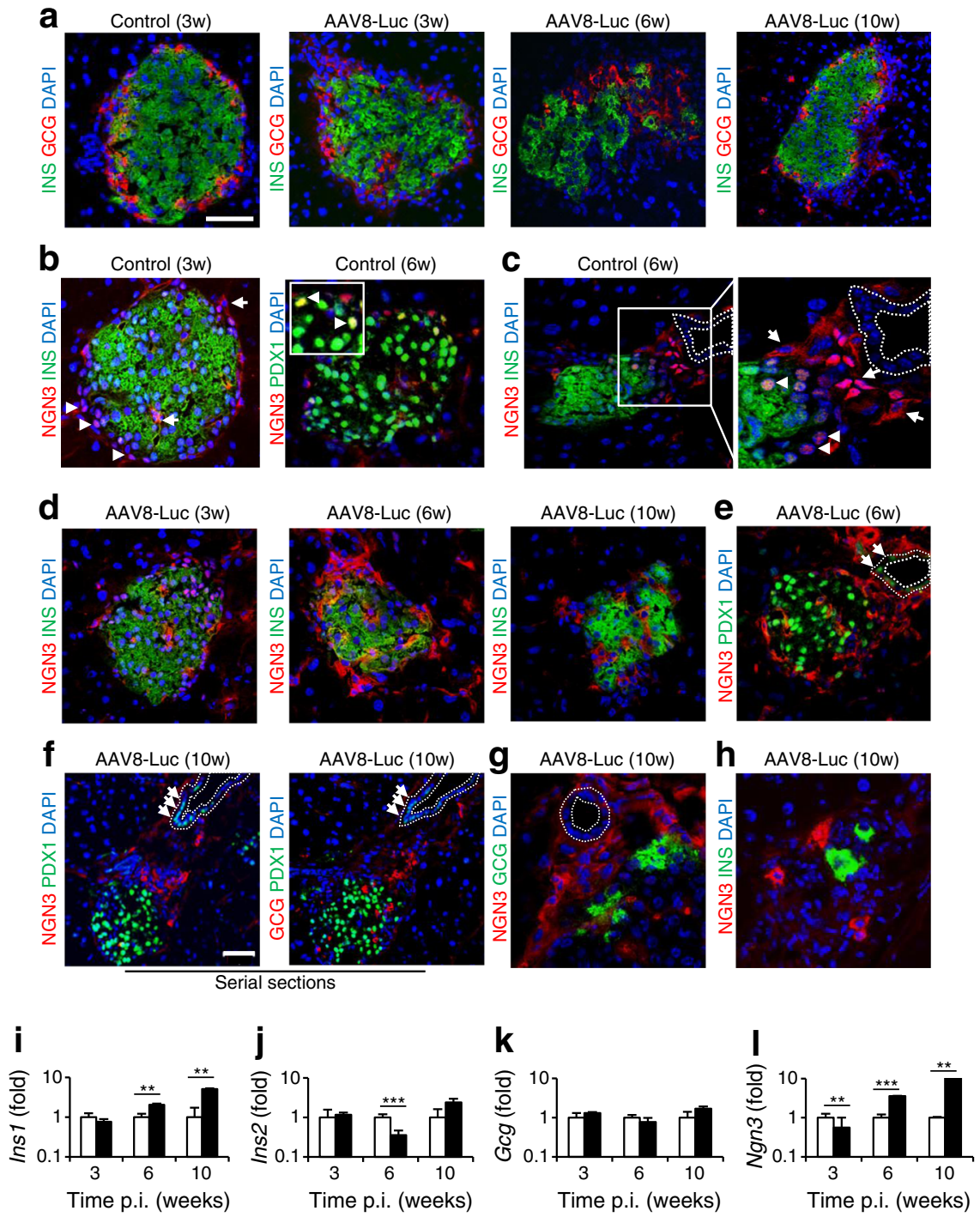

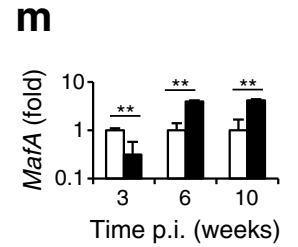

n

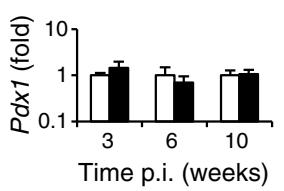

Fig. 4 Displacement of glucagon-positive cells and activation of NGN3positive cells following immunological beta cell damage. (a) The location of insulin (INS)-positive and glucagon (GCG)-positive cells was determined by staining control and immunologically damaged islets with antiinsulin (green) and anti-glucagon (red) antibodies at 3,6 and 10 weeks p.i. (b) NGN3 expression was detected using an anti-NGN3 antibody in agematched control islets at 3 and 6 weeks p.i.. Note cytoplasmic (arrows) and nuclear localisation (arrowheads) in normal islets. (c) Immunostaining demonstrated both nuclear (arrowheads) and cytoplasmic (arrow) NGN3 expression in age-matched control islets near ducts. (d) NGN3 expression was analysed in islets from AAV8-Luc-transduced mice at 3, 6 and 10 weeks p.i. Insulin expression was detected using an anti-insulin antibody. (e) Islets from AAV8-Luc-transduced mice at 6 weeks p.i. were analysed for NGN3 and PDX1 (green) expression. Note induction of
0

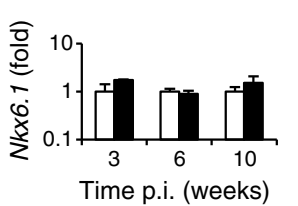

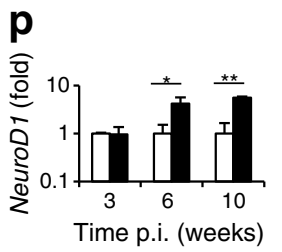

PDX1 expression in ductal lining cells (arrows). (f) Representative islets showing NGN3, PDX1 and glucagon localisation after immune response. Nuclear PDX1-positive cells (arrows) were identified in ductal lining cells. (g) Immunostaining of the region between recovering islets and ducts with anti-NGN3 and glucagon antibodies. (h) A representative image of single insulin-positive cells (green) identified at 10 weeks p.i. is shown with anti-NGN3 co-staining (red). (a-h) Dashed lines correspond to ductal epithelium. Scale bars, $50 \mu \mathrm{m}$; w, weeks. (i-p) Quantitative RT-PCR analysis of pancreatic transcripts for common islet- and beta cell-associated factors. Results show AAV8-Luc transcripts (black bars) relative to age-matched control (white bars) pancreas, set as 1 ; logarithmic scale, base 10. Data are presented as means \pm SEM, representing four mice per group per time point. ${ }^{*} p<0.05,{ }^{*} p<0.01$, $* * * p<0.001 ;$ INS, insulin 
cells around islets (ESM Fig. 8a, centre and right panels). Although pancreatic stellate cells have been reported to express NES and glial fibrillary acidic protein (GFAP) during pancreatic inflammation [27], we found NES and GFAP staining in the same location, but not necessarily in the same cells (ESM Fig. 8a, right panel). Quantitative RT-PCR analysis showed increased levels of Nes transcripts in inflamed pancreas at 3 weeks p.i. (ESM Fig. 8b). Recruitment of CD34-positive cells was evident at the same time point (ESM Fig. 8c). Indeed, many NES-positive cells in and around damaged islets were also positive for CD34 and endoglin (CD105; ESM Fig. 8d), suggesting that NES/CD34 double-positive cells are vascular endothelial cells. Some NES-positive cells did not express CD34 or CD105 (ESM Fig. 8d). Similar to the biased distribution of NGN3- and glucagon-positive cells during islet recovery, more NES-positive cells were found between recovering islets and ducts at 10 weeks p.i. (ESM Fig. 8e), although the NES-positive cells were distinct from glucagon- and NGN3-expressing cells (ESM Fig. 8e, f). We also induced immunological beta cell damage in a NES-positive cell lineage tracing mouse model (ESM Fig. 8g). A subset of acinar cells, as well as vascular endothelial-like cells, was labelled with membrane EmGFP (mGFP) in control and insulitis-induced mice (ESM Fig. 8g). There were no mGFP-positive beta cells, thus ruling out a direct contribution of NES-positive cells to beta cell development and regeneration.

Induced immunological beta cell destruction leads to beta cell regeneration in aged mice One-year-old mice $(n=6$ per treatment) were i.p. injected with AAV8-Luc, and monitored for luciferase expression. Luciferase signals in the pancreas reached a peak at around 3 weeks p.i., followed by a marked decline by 10 weeks p.i. (ESM Fig. 9), which correlated with notable immune infiltration in treated islets (ESM Fig. 10a). Upon immunological beta cell destruction in aged mice, similar trends were observed in terms of induction of NES- or NGN3-positive cells (Fig. 5a, ESM Fig. 10b), reduced beta cell mass (Fig. 5b) and increased insulin-positive cell
Fig. 5 Induced immunological beta cell destruction leads to beta cell regeneration in aged mice. (a) Mice received AAV8-Luc vector through i.p. injection. Immunohistochemistry was performed at 6 and 10 weeks p.i. Scale bars, $50 \mu \mathrm{m}$. (b) Percentage insulin-positive area was determined as described in Fig. 3b. (c) Beta cell proliferation was determined as described in Fig. 3c. Images of islets at 6 weeks are shown. Beta cell proliferation was determined by quantification of total insulin- and Ki67-positive cells in five random islets from age-matched controls $(n=4$ per time point) and AAV8-Luctransduced mice ( $n=4$ per time point). ${ }^{*} p<0.05$ vs control. (d) A representative islet in AAV8-Luctreated mice at 10 weeks p.i., showing a dense population of Ki67-positive cells with a few isolated insulin-positive and Ki67positive cells. (e) Fasting blood glucose levels were determined over the 10 week period for treated $(n=4)$ and control mice $(n=4)$.

${ }^{* *} p<0.01$ vs control. (f) Glucose tolerance tests were conducted at 5 weeks p.i. when vector-treated mice showed higher fasting blood glucose levels (see Fig. 5e). GCG, glucagon; INS, insulin; (b, c, e, f) white bars/squares, saline controls; black bars/squares, AAV8-Luc treatment
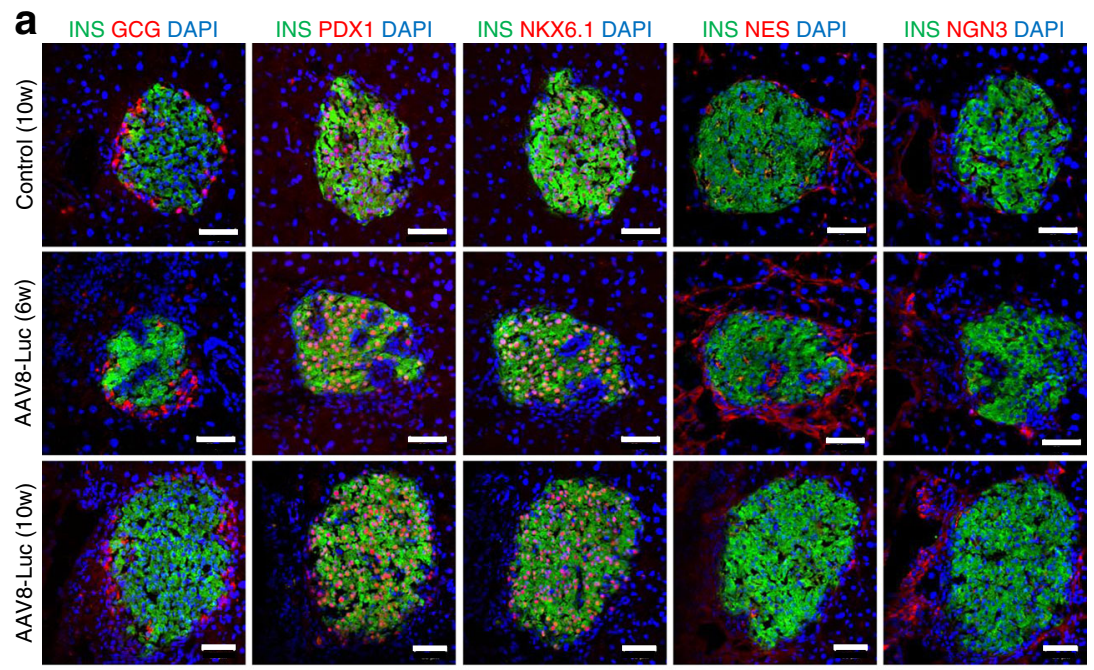

b

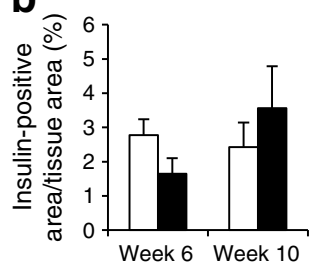

C
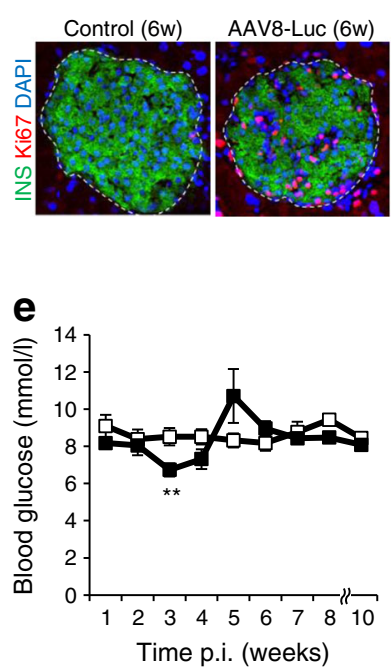

d

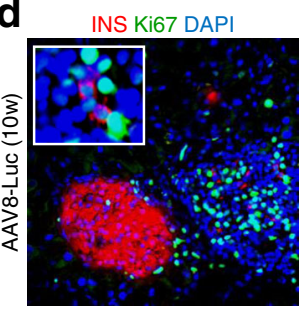

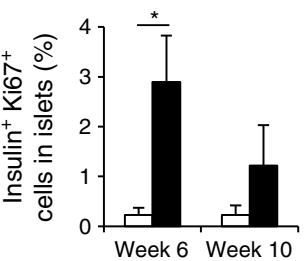

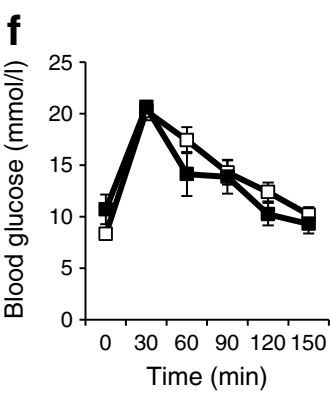


proliferation (Fig. 5c). Additionally, groups of single insulinexpressing cells, some expressing Ki67, were observed at 10 weeks p.i. (Fig. 5d, ESM Fig. 11). Although these observations demonstrate the robust regenerative capacity of beta cells following immunological damage in aged mice, many recovering islets initially exhibited notable scarring (Fig. 5a, ESM Fig. 10), and there were temporary fluctuations in blood glucose levels (Fig. 5e, f). Those observations suggest that beta cell repair responses are slower in aged mice than in their younger counterparts.

\section{Discussion}

We used a beta cell-targeting AAV8 vector to induce singleround expression of a target antigen, firefly luciferase, in preexisting beta cells. In this model, newly regenerated beta cells do not express the target antigen, thus allowing visualisation of beta cell recovery following immunological beta cell destruction. Luciferase expression also allowed non-invasive live imaging of beta cells under immunological attack. Since AAV vectors have broad host ranges, our 'hit-and-run' antigen transduction system can be used to induce immunological beta cell injury in various animal species, including rodents, large animal models and non-human primates, with particular genetic backgrounds and ageing status. It is plausible that immunological beta cell destruction in mice with a high-risk type 1 diabetes genetic background can lead to full-blown autoimmune diabetes. In addition, the use of different AAV serotype vectors, such as AAV8 and AAV9, with beta cell tropism $[15,16,28,29]$ would allow multiple rounds of vector administration to beta cells, providing an opportunity to assess the contribution of environmental triggers and the long-term influence of multiple asymptomatic immune events on beta cell proliferative capability in animal models.

Beta cell proliferation is increasingly recognised as a primary pathway for beta cell expansion during normal postnatal development, pregnancy, or following partial pancreatectomy or partial beta cell ablation [30-33]. Here, we found robust beta cell proliferation following immunological beta cell destruction in young mice. Increased beta cell proliferation was evident as early as 3 weeks p.i. (Fig. 3c, d), when luciferasetargeted humoral, but not cellular, immunity was detectable (Fig. 2e), and until replenishment of the beta cell mass at 10 weeks p.i. Although their beta cell mass recovery was slower, aged mice also demonstrated significantly increased beta cell proliferation after immunological beta cell destruction. Our data therefore suggest that beta cell proliferation is the primary mode of beta cell regeneration following immunological beta cell destruction, consistent with previous studies demonstrating increased beta cell proliferation in recentonset type 1 diabetic patients $[4,20]$.
Various cell types have been proposed as potential progenitors of beta cells [34]. For instance, partial duct ligation (PDL) induces PDX1 expression in duct-lining cells and mobilises NGN3-positive cells, which can give rise to beta cells $[35,36]$. The conversion of adult alpha cells to beta cells has also been demonstrated after extreme beta cell loss [22]. In our study, immune-mediated beta cell destruction frequently led to alpha cell mass increase and displacement, confirming previous studies in type 1 diabetic patients or in NOD mice with immunological beta cell damage [4, 23]. Groups of glucagon-positive cells were also found between recovering islets and adjacent ducts at 10 weeks p.i. Similarly, increased numbers of cytoplasmic NGN3-positive cells were found around damaged and recovering islets, with PDX1 induction in ductal cells near to recovering islets. At present, it is not known whether those cells can directly differentiate into beta cells. Recently, Xiao et al (2013) showed increased NGN3 expression in proliferating ductal cells and existing beta cells after PDL but that these cells do not directly contribute to beta cell neogenesis [30]. Considering that beta cell proliferation starts at 3 weeks p.i. and notable recruitment and activation of alpha cells and cytoplasmic NGN3-positive cells is initiated at later time points, it is less likely that alpha cells and cytoplasmic NGN3-positive cells play primary roles in beta cell regeneration, at least in the early stage of beta cell regeneration after immunological beta cell destruction. Since beta cell injury can activate alpha cells to produce GLP-1, a growth and survival factor for beta cells [23], it is plausible that alpha cells indirectly support beta cell recovery through a paracrine action. Combinations of genetic lineage tracing studies using our AAV8-mIP-Luc vector-mediated immunological destruction model would reveal whether specific cell types act as beta cell progenitors following immunological beta cell destruction.

NES-positive cells were initially recognised as multipotential stem cells in the adult pancreas [26]. Immunological beta cell damage induced the rapid expansion, and potentially the recruitment, of NES-positive cells in and around damaged islets. Nevertheless, our lineage tracing experiment demonstrated that NES-positive cells contribute to exocrine and microvasculature cells, but not to beta cells, supporting a previous report [37]. Our preliminary data using the AAV8mIP2-Luc vector in NES knockout mice suggest roles for NES-positive endothelial cells in the recruitment of CD11bpositive immune cells to damaged islets and in the clearance of apoptotic beta cells from damaged islets (J. M. Tonne and Y. Ikeda, unpublished data). Further study should shed light on the role of the NES-positive cells in type 1 diabetes pathogenesis.

Another notable finding is that immunological beta cell destruction induces responses reminiscent of those reported in the PDL mouse model $[35,36]$, such as induction of ductal PDX1 expression, activation of NGN3-positive cells and 
recruitment of NES-positive cells. It is conceivable that immunological beta cell damage and physical injury to the pancreas activate a conserved beta cell recovery process.

In conclusion, we have demonstrated that the beta celltargeted AAV8 vector can induce immunological beta cell destruction. Our results validate the use of this system as a tool to induce single-round beta cell destruction as well as visualising beta cell regeneration after immunological ablation. Our model provides a valuable platform to characterise beta cell regeneration after immunological destruction in various animal models with different genetic backgrounds and ageing status.

Acknowledgements We would like to thank R. Kulkarni, Joslin Diabetes Center, Boston, Massachusetts, USA, for helpful suggestions. The AAV8 capsid-expressing plasmid pRC-2/8 was kindly provided by $\mathrm{J}$. Wilson (University of Pennsylvania, Philadelphia, PA, USA). We also thank J. E. Tarara and D. M. Deal (Mayo Clinic, Rochester, MN, USA) for extensive technical aid in confocal image acquisition and analysis.

Funding This work was supported by Mayo Foundation, Eisenberg Stem Cell Trust, Decade of Discovery Minnesota Partnership Grant (YI), Mayo Center for Regenerative Medicine (YI and TS) and the National Institutes of Health (DK085516; YK).

Duality of interest statement The authors declare that there is no duality of interest associated with this manuscript.

Author contributions JMT designed and directed the study, acquired and analysed data, and wrote and edited the manuscript; TS acquired data and edited the manuscript; MMG and MEK acquired and analysed data, and edited the manuscript; MAB and YCK designed the study and edited the manuscript; YI designed and directed the study, analysed data, and wrote and edited the manuscript. All authors contributed to revising the article and all approved the final version. YI is responsible for the integrity of the work as a whole.

Open Access This article is distributed under the terms of the Creative Commons Attribution License which permits any use, distribution, and reproduction in any medium, provided the original author(s) and the source are credited.

\section{References}

1. Daneman D (2006) Type 1 diabetes. Lancet 367:847-858

2. van Belle TL, Coppieters KT, von Herrath MG (2011) Type 1 diabetes: etiology, immunology, and therapeutic strategies. Physiol Rev 91:79-118

3. Zhao HL (2006) Comment on: Meier JJ, Lin JC, Butler AE, Galasso R, Martinez DS, Butler PC (2006) Direct evidence of attempted beta cell regeneration in an 89-year-old patient with recent-onset type 1 diabetes. Diabetologia 49:1838-1844. Diabetologia 49: 2801-2802; author reply 2803-2804

4. Willcox A, Richardson SJ, Bone AJ, Foulis AK, Morgan NG (2010) Evidence of increased islet cell proliferation in patients with recentonset type 1 diabetes. Diabetologia 53:2020-2028

5. Karges B, Durinovic-Bello I, Heinze E, Boehm BO, Debatin KM, Karges W (2004) Complete long-term recovery of beta-cell function in autoimmune type 1 diabetes after insulin treatment. Diabetes Care 27:1207-1208

6. Sherry NA, Kushner JA, Glandt M, Kitamura T, Brillantes AM, Herold KC (2006) Effects of autoimmunity and immune therapy on beta-cell turnover in type 1 diabetes. Diabetes 55:3238-3245

7. Sreenan S, Pick AJ, Levisetti M, Baldwin AC, Pugh W, Polonsky KS (1999) Increased beta-cell proliferation and reduced mass before diabetes onset in the nonobese diabetic mouse. Diabetes 48:989996

8. Zorina TD, Subbotin VM, Bertera S et al (2003) Recovery of the endogenous beta cell function in the NOD model of autoimmune diabetes. Stem Cells 21:377-388

9. Kodama S, Kuhtreiber W, Fujimura S, Dale EA, Faustman DL (2003) Islet regeneration during the reversal of autoimmune diabetes in NOD mice. Science 302:1223-1227

10. Suri A, Calderon B, Esparza TJ, Frederick K, Bittner P, Unanue ER (2006) Immunological reversal of autoimmune diabetes without hematopoietic replacement of beta cells. Science 311:1778-1780

11. Chong AS, Shen J, Tao J et al (2006) Reversal of diabetes in nonobese diabetic mice without spleen cell-derived beta cell regeneration. Science 311:1774-1775

12. Nishio J, Gaglia JL, Turvey SE, Campbell C, Benoist C, Mathis D (2006) Islet recovery and reversal of murine type 1 diabetes in the absence of any infused spleen cell contribution. Science 311:1775-1778

13. Nakai H, Yant SR, Storm TA, Fuess S, Meuse L, Kay MA (2001) Extrachromosomal recombinant adeno-associated virus vector genomes are primarily responsible for stable liver transduction in vivo. J Virol 75:6969-6976

14. Cataliotti A, Tonne JM, Bellavia D et al (2011) Long-term cardiac pro-B-type natriuretic peptide gene delivery prevents the development of hypertensive heart disease in spontaneously hypertensive rats. Circulation 123:1297-1305

15. Wang Z, Zhu T, Rehman KK et al (2006) Widespread and stable pancreatic gene transfer by adeno-associated virus vectors via different routes. Diabetes 55:875-884

16. Tonne JM, Sakuma T, Deeds MC et al (2013) Global gene expression profiling of pancreatic islets in mice during streptozotocininduced beta-cell damage and pancreatic Glp-1 gene therapy. Dis Model Mech 6:1236-1245

17. Falk K, Rotzschke O, Faath S et al (1993) Both human and mouse cells expressing $\mathrm{H}-2 \mathrm{~Kb}$ and ovalbumin process the same peptide, SIINFEKL. Cell Immunol 150:447-452

18. Limberis MP, Bell CL, Wilson JM (2009) Identification of the murine firefly luciferase-specific CD8 T cell epitopes. Gene Ther 16:441447

19. Campbell-Thompson ML, Atkinson MA, Butler AE et al (2013) The diagnosis of insulitis in human type 1 diabetes. Diabetologia 56: 2541-2543

20. Meier JJ, Nauck MA, Butler PC (2006) Comment to: Patti ME, McMahon G, Mun EC et al. (2005) Severe hypoglycaemia postgastric bypass requiring partial pancreatectomy: evidence for inappropriate insulin secretion and pancreatic islet hyperplasia. Diabetologia 48:2236-2240. Diabetologia 49: 607-608; author reply 609-610

21. Gerich JE, Lorenzi M, Karam JH, Schneider V, Forsham PH (1975) Abnormal pancreatic glucagon secretion and postprandial hyperglycemia in diabetes mellitus. JAMA 234:159-165

22. Thorel F, Nepote V, Avril I et al (2010) Conversion of adult pancreatic alpha-cells to beta-cells after extreme beta-cell loss. Nature 464: $1149-1154$

23. Habener JF, Stanojevic V (2012) Alpha-cell role in beta-cell generation and regeneration. Islets 4:188-198

24. Lendahl U, Zimmerman LB, McKay RD (1990) CNS stem cells express a new class of intermediate filament protein. Cell 60:585-595 
25. Civin CI, Strauss LC, Brovall C, Fackler MJ, Schwartz JF, Shaper JH (1984) Antigenic analysis of hematopoiesis. III. A hematopoietic progenitor cell surface antigen defined by a monoclonal antibody raised against KG-1a cells. J Immunol 133:157-165

26. Zulewski H, Abraham EJ, Gerlach MJ et al (2001) Multipotential nestin-positive stem cells isolated from adult pancreatic islets differentiate ex vivo into pancreatic endocrine, exocrine, and hepatic phenotypes. Diabetes 50:521-533

27. Lardon J, Rooman I, Bouwens L (2002) Nestin expression in pancreatic stellate cells and angiogenic endothelial cells. Histochem Cell Biol 117:535-540

28. Jimenez V, Ayuso E, Mallol C et al (2011) In vivo genetic engineering of murine pancreatic beta cells mediated by single-stranded adeno-associated viral vectors of serotypes 6,8 and 9 . Diabetologia 54:1075-1086

29. Xiao X, Gaffar I, Guo P et al (2014) M2 macrophages promote betacell proliferation by up-regulation of SMAD7. Proc Natl Acad Sci U S A 111:E1211-E1220

30. Xiao X, Chen Z, Shiota C et al (2013) No evidence for beta cell neogenesis in murine adult pancreas. J Clin Invest 123:2207-2217
31. Dor Y, Brown J, Martinez OI, Melton DA (2004) Adult pancreatic beta-cells are formed by self-duplication rather than stem-cell differentiation. Nature 429:41-46

32. Teta M, Rankin MM, Long SY, Stein GM, Kushner JA (2007) Growth and regeneration of adult beta cells does not involve specialized progenitors. Dev Cell 12:817-826

33. Nir T, Melton DA, Dor Y (2007) Recovery from diabetes in mice by beta cell regeneration. J Clin Invest 117:2553-2561

34. Bonner-Weir S, Li WC, Ouziel-Yahalom L, Guo L, Weir GC, Sharma A (2010) Beta-cell growth and regeneration: replication is only part of the story. Diabetes 59:2340-2348

35. Xu X, D’Hoker J, Stange G et al (2008) Beta cells can be generated from endogenous progenitors in injured adult mouse pancreas. Cell 132:197-207

36. Van de Casteele M, Leuckx G, Baeyens L et al (2013) Neurogenin 3+ cells contribute to beta-cell neogenesis and proliferation in injured adult mouse pancreas. Cell Death Dis 4:e523

37. Treutelaar MK, Skidmore JM, Dias-Leme CL et al (2003) Nestinlineage cells contribute to the microvasculature but not endocrine cells of the islet. Diabetes 52:2503-2512 